\title{
Improvement of the Performance of Microstrip Patch Antenna
}

\author{
Snehi Saraswati \\ Sam Higginbottom Institute of Agriculture, \\ Technology and Sciences \\ Department of Electronics and Communication \\ Engineering \\ Naini, Allahabad - 221007, \\ India
}

\begin{abstract}
The Microstrip patch antennas have the properties of low cost fabrication and least profile. This Antenna is more properly used now a day due to its light weight, low volume and easy to install on the rigid surface. In this paper, the design of the microstrip patch antennas are considered. Using the fractal technology,on Sierpinski Carpet antenna, return loss measurement, radiation pattern etc. have been investigated. The discussions and conclusions of this paper are helpful in designing and improving the performance of microstrip patch antennas as per need of the society.
\end{abstract}

\section{General Terms}

Microstrip line feed, coaxial feed, substrate permittivity, dielectric substrate, fractal technology, radiation pattern and return loss measurement

\section{Keywords}

Microstip Patch Antenna, low profile antenna, leaky waves, bandwidth, feeding techniques and Sierpinski carpet antenna

\section{INTRODUCTION}

In ancient time sound and voice played very important role in communication among human beings. Consequently drums, visual methods such as signal flags and smoke signals were used for slightly more distance communication as per requirement of the society. The light portion of the electromagnetic spectrum is utilized during these optical communication devices. Radio, one of the most important devices for communication, is generally used outside the visible region.

Presently, there is a need in the development of personal communication service (PCS) devices which are integrated parts of the society. Cellular phones and wireless local area networks (WLAN) are considered in the range of the personal communication services (PCS). It is remarkable to note that the cellular phones incorporate digital cameras and web browsing. PCS and WLAN are linked together and their applications have wide range. People use laptop and television without any wire connectivity. A WLAN is a special type of communication network which is flexible in nature and generally used for extension for a wired LAN in a building. They are primarily used in the following ways/manners:

1. In industrial sectors where employees are more in number

2. In temporary locations and

\author{
Neelesh Agrawal \\ Sam Higginbottom Institute of Agriculture, \\ Technology and Sciences \\ Department of Electronics and Communication \\ Engineering \\ Naini, Allahabad - 221007 \\ India
}

3. where cabling may hinder the installation of wired LAN.

Since the technology has been become more affordable therefore tremendously increasing more wireless LANs are being setup in homes and offices. Researchers are predicting that almost all notebooks will contain integrated WLAN in the near future. Faster technology and higher transfer rates are the needs of progress and expansion of the wireless communication for modern society. WLAN technology is playing a key role in wireless data transmission. Research and development activities as well as ongoing wireless LAN standardization tangent transfer rates greater than $100 \mathrm{Mbps}$ cellular network operators exploit WLAN technology due to this cellular data networkings are being in progress.

The development of efficient broadband antennas is capable for the popularity of indoor wireless LAN. These systems are used frequently in residential and official areas due to low profile, low cost and highly effective and efficient. The electromagnetic spectrum is a natural resource for the communication while the role of the antenna is considered as instrumental in harnessing this type of resources.

The communication systems gradually converted into wireless communication systems. An antenna is considered as a very crucial part of a wireless communication system. The design of the antenna is totally responsible for overall performance of the wireless communication system. Thus for a successful operation of this system, the used antenna should be properly designed and perfectly matched with operating frequency. A micostrip patch antenna provides several advantages over traditional antennas because it offers a low profile such as thin and easily manufacturability.

In modern society, Microstrip patch antennas are more popularly used for communication due to its following advantages:

1. Light weight

2. Low volume

3. Compatibility with integrated circuits

4. Easy to install on the rigid surface

5. Low cost

6. Minimum disturbances

7. Easy to fabrication and

8. Highly reliable in hazardous conditions like speed, atmospheric pressure etc 
Recently microstrip antennas are designed to operate in dualband as well as multi-band application that may be circular or dual polarization. These antennas are helpful in different handheld communicating devices. A dielectric substrate having fixed dielectric constant is a main component of a simple microstrip patch antenna.

A ground plane is present on one side of a dielectric substrate and radiating patch is found on another side of that substrate.

Generally, the metallic patch takes the following geometrical shapes.

1. Square

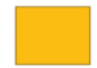

\section{Rectangular}

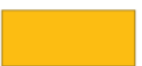

3. Circular

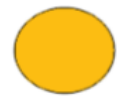

4. Triangular

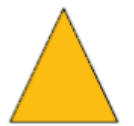

5. $\quad$ Ring

6. Elliptical etc
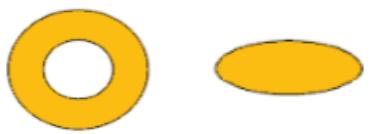

The dimension of the patch depends on the resonant frequency of antenna.

Microstrip patch antennas are having narrow bandwidth. For the practical applications and necessaries, there are needs of enhancement of bandwidth of the antenna. Thus the several different approaches are utilized for increasing the bandwidth. The defected Ground Structure (DGS) is one of the approaches. The mobile handheld communicating devices require antennas of smaller size. These devices are the applications of microstrip antennas in communication systems. In the last few years, several advance tools have been designed to improve the functioning of compact micro strip patch antennas.

In ground plane of patch antenna, some defected shape is introduced and the shielded current distribution depends on shape, size and the dimension of the defect during the communication. This process is one of the explanations of DGS (Defected Ground Structure) Due to disturbance at the shielded current distribution, current flow of the antenna and input impedance have been affected. This is helpful in controlling the excitation and electromagnetic waves which propagate through the substrate layer.
Electromagnetic band-gap structure have their unique electromagnetic features i.e. forbidden band gap and in phase reflection. These are widely used in antenna and microwave element designs. The electromagnetic band gap (EBG) is originated from a new artificial electromagnetic material known as photonic crystal in the antenna community. Presently an electromagnetic band gap structure (EBG) is composed of cyclical array of metal or dielectric elements. This is considered as one special electromagnetic material.

EBG structures have following properties:

1. Frequency bad gap and

2. Phase reflection.

These two properties are considered mainly in designs of microwave antenna. This is an application of EBG structure.

The design of microstrip patch antenna is obtained by surface wave-suppression. This design is with high gain, suppress high order harmonic and depress the mutual coupling of plannar antennas. It is remarkable to note that the applications of the inphase reflection property are as following:

1. To raise the antenna gain as a reflection plate, Inhibit the rear lobe and

2. To design low profile antenna.

Recently, the researchers are concentrating on the mushroomlike EBG structure because its compactness property is more applicable than other EBG structures.

There are a number of favourable applications of microstrip patch antennas in wireless communication field due to their small size, ease of integration, light weight and low price. Miniaturization is the essential antennas design requirements which enable the revolutionary development of these communication systems.

The researchers are trying their best to make the antenna small in size and having low cast. The raising the permittivity of the substrate is responsible to deteriorate the performance of microstrip antennas.

\section{AIM AND OBJECTIVE}

Microstrip patch antenna is generally used to transfer the onboard parameters of the article to the ground in case of operating conditions. One of the objectives of this paper is to design and fabricate an inset-fed rectangular Microstrip patch Antenna.

The effects of antenna dimensions Length (L), Width (W), substrate parameters, substrate thickness (t), relative dielectric constant $\left(\mathrm{e}_{\mathrm{r}}\right)$ and role of radiation parameters of bandwidth and beam-width are to be studied.

\section{LITERATURE REVIEW}

A microstrip antenna is suitable for conducting patch on a ground plane which is separated by dielectric substrate. During 1970, the revolution in electronic circuit miniaturization and large-scale integration took place. After this, gradually the development in microstrip antenna is in progress. Several researchers have described the radiation from the ground plane for many configurations by a dielectric substrate. Previously, a low profile flush mounted antennas were used on rockets and missiles which indicated the practical uses of several antenna system. Various mathematical models for antennas were developed and their applications are extended to other related fields. 
In microstrip antenna, the substrate material plays an important role in determining the size and bandwidth of the antenna. The decreasing the dielectric constant increases the bandwidth but increases in size. In case of increasing the dielectric constant, the size of the antenna decreases but lowers the bandwidth and the efficiency of the antenna.

Sakshi kappor[1] has observed that the antenna is feed with the help of microstrip feeding technique and IE3D software. The signal band width of $2 \mathrm{GHz}$ for the band of $4-6 \mathrm{GHz}$ is generally investigated in this antenna. Working in the same direction, The researchers are proposing the antenna useful for IEEE 802.11 WLAN standards in the 5.2/5.8 GHz band and WiMAX standards in the $5.5 / 5.8 \mathrm{GHz}$ band and WiMAX standards in the $5.5 \mathrm{GHz}$ band and for more comfortable range which is as per need of the modern society.

Gupta, Nakhate and Shandilya [2] have seen that defected L shaped slot and rectangular parasitic patches are not suitable for antennas because the diagonal cuts at top corners may increase the bandwidth for the first and second reasonant frequencies. In case of diagonal cut at optimum value, the return losses of $-17 \mathrm{~dB}$ and $-30 \mathrm{~dB}$ may be achieved.

Recently, several researchers, using High frequency Simulation Software (HFSS) at $2.45 \mathrm{GHz}$ frequency antenna in the form of rectangular microstrip patch antenna has been simulated. This antenna is fed by Quarter Transformer feeding. The rectangular patch antennas having swastika shaped DGS (Defected Ground Structure) have achieved gain of $7 \mathrm{~dB}$ (approximately). This type of antennas are used preferably than traditional (or conventional) antennas because there are improved returning loss, VSWR (voltage standing wave ratio) and bandwidth.

Arya and Kartikeyan [3] have observed that DGS (Defected Ground Structure) has applications in microwave oscillators, filter design, microwave amplifiers etc. DGS is generally used in the microstrip antennas design for following advantages:

1. Antennas size reduction

2. Mutual coupling reduction

3. Harmonic suppression and

4. Cross polarization reduction in antenna array etc.

Working in this direction, Ahuja, Khanna and Kaur [4] have studied microstrip patch antenna for GSM (Global System for Mobile Communication) and Wi-Max application. This antenna has promising characteristics for WLAN (Wireless Local Area Network), Wi-Max and satellite applications.

Presently, Dinakaran et.al[5] have designed rectangular patch antenna for $\mathrm{Wi}-\mathrm{Fi}$ applications frequency is very closed to the specified frequency band feasible to $\mathrm{Wi}-\mathrm{Fi}$ applications . Kapadia and Patel [6] have studied Fractal antenna .

\section{ROLE OF WAVES ON MICROSTRIP}

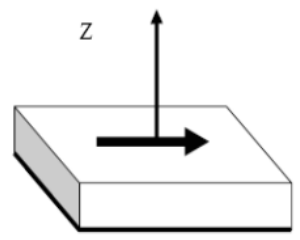

Fig 4.1 Hertz dipole on a microstrip substrate

Let us consider a Hertz dipole a point current source on a microstrip substrate. This is helpful in understanding the mechanisms of transmission and radiation in a microstrip. The electromagnetic waves are radiated from this source. There is a major role of the direction of the transmitted waves on the basis of this direction, They can be distincted into following categories:
a. Surface waves
b. Leaky waves and
c. Guided waves

There are different behaviour of these waves.

\subsection{Surface Waves}

The waves transmit with the elevation angles $\theta$ such that $\pi / 2 \leq \theta \leq \pi \arcsin (1 / \sqrt{\varepsilon})$ and meets the ground plane. It reflects them to meet the dielectric-to-air boundary. In this process, the dielectric-to-air boundary reflects them to total reflection condition. As similar to the modes in metallic waveguide, the magnitude of the field amplitudes is responsible for some particular incidence angles which leads to the excitation of a discrete set of surface wave modes.

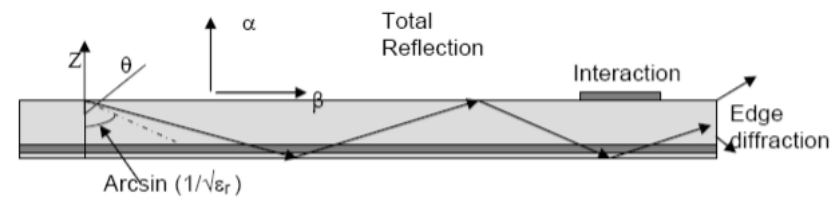

Figure 4.1.1 Surface Wave

\subsection{Leaky Waves}

The waves direct downword with angles $\theta$ such that $\pi$ $\arcsin \left(1 / \sqrt{ }_{\mathrm{r}}\right)<\theta<\pi$ then they are reflected by the ground plane and partially by the dielectric-to-air boundary. Thus, waves leak from the substrate into the air. Due to this character, they are known as leaky waves which contribute to the radiation. Generally, leaky waves are non-uniform plane waves having attenuation direction $\alpha$ pointed downword which may be odd. The amplitude of the waves increases when one of them moves away from the dielectric surface. The field amplitude increases as one moves away from the substrate. This is possible due to radiation of waves from a point with larger signal amplitude. Due to finite structure and existence of locally divergent behaviour, the wave vanishes abruptly in case of crossing its trajectory which is shown in figure of locally divergent behaviour, the wave vanishes abruptly in case of crossing its trajectory which is shown in figure

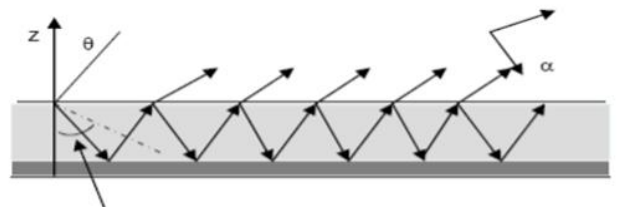

Figure 4.2.2 Leaky wave

$$
\operatorname{Arcsin}\left(\frac{1}{\sqrt{\varepsilon_{r}}}\right)
$$

To obtain a larger gain, a more general structure has several layers of different dielectrics and the leaky waves are helpful in increasing the apparent size of the antenna as per need of the society. This happens for favourable stacking arrangements at a suitable frequency. In some multilayer structures, the leaky waves are not excited. 


\subsection{Guided Waves}

A metal layer is added on the top of the substrate which introduces an additional reflecting boundary. Thus, the geometry of the antenna has been modified. The dielectric-toair boundary is generally located under the upper conductor bounce back and forth on the boundaries of the metal. The waves are directed into the dielectric-to-air boundary. This forms a parallel plate waveguide. Only for some particular values of the incident angle, the waves exist in the metallic guide. In this way, a discrete set of waveguide modes is formed. The guided waves help to the transmission lines and circuits. During this process, the electromagnetic fields are concentrated in the layer below the upper conductor. This kind of electromagnetic energy is not favourable for patch antenna because this works like resonators having a limited frequency bandwidth

\section{CHARACTERISTICS OF ANTENNA}

Really an antenna is a device/method to radiate and receive radiated electromagnetic waves. Keeping following characteristics, the suitable antenna are selected for the need of the modern society:
1. Antenna radiation patterns
2. Directivity
3. Power gain
4. Polarization

\section{DISADVANTAGE OF MICROSTRIP PATCH ANTENNAS}

There are some disadvantages of microstrip patch antennas as compared to conventional antennas. Some of their disadvantages are as following:

1. Low gain

2. Low efficiency

3. Low power handling capacity

4. Narrow bandwidth

5. Extraneous radiation from feeds and junction

6. Surface wave excitation

7. Poor and fire radiator except tapered slot antennas

\section{FOUNDATIONS FOR THE DESIGN OF MICROSTRIP PATCH ANTENNAS}

Conventional antennas

Let us first consider a traditional/conventional patch antenna.



Fig 7.1 Conventional antenna dimensions
This reference antenna works at $1.8 \mathrm{GHz}$ so that the centre frequency of the antenna may be covered with the band gap region of mushroom-like EBG (Electromagnetic band gap) structure for remaining its compact size, the patch is fabricated with a relative permittivity of 10.2 on the ground plane. The resonant frequency of this antenna is at $1.8 \mathrm{GHz}$ with area of the patch $=L \times W=24.8 \times 38.3 \mathrm{~mm}^{2}$ and the feed location $\mathrm{L}_{1}=3.34 \mathrm{~mm}$ where $\mathrm{L}_{1}$ is the distance between the feed and the centre of the patch.

One side of a dielectric substrate, a micro strip patch antenna is a radiating patch and it has a ground plane on the underside. The EM (Electromagnetic) waves fringe off the top patch into the substrate. They reflect off the ground plane and consequently radiates out into the air. Due to fringing field between the ground and patch, the radiation takes place. The permittivity $\left(e_{r}\right)$ of the dielectric influences the radiation efficiency of the patch antenna. Generally, low $\mathrm{e}_{\mathrm{r}}$, a thick dielectric and low insertion loss are preferred for the purposes of broadband and large efficiency.

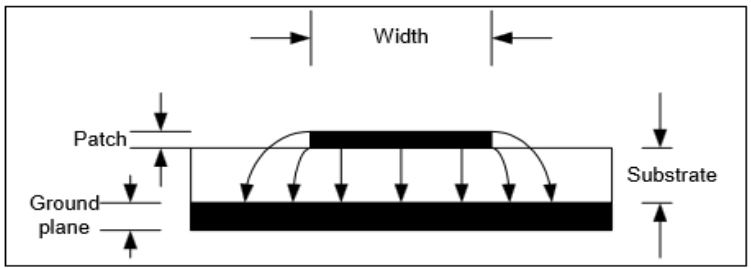

Fig7.2 Operation of a microstrip patch antenna

\subsection{Polarization Types}

The polarization of waves radiated by the antenna in a particular direction is generally dependant on the technique of the feeding. It is in the direction of maximum radiation when direction is not specified. There are two widely used polarization types.

\subsubsection{Linear Polarization}

A slot antenna is very useful and it is the simplest form and counter part of a linearly polarized antenna. In this case, The $\mathrm{E}$ field is oriented perpendicular to its length dimension. The microstrip patches are different variations and there is the radiation due to linear polarization. The operations of a linearly polarized wave radiating perpendicular to the patch plane are shown in following fig(7.3)

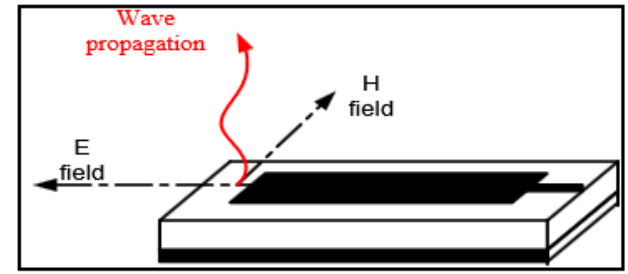

Fig. 7.3 Linear polarization

\subsubsection{Circular Polarization}

The orthogonal fed signal input is the main factor of the circular polarization(C.P) It is observed that if two signals of equal amplitude but 90 degree phase difference are shifted then the resulting wave is generally polarized in circular form. In case of anticlockwise rotation of the wave, there is Left hand circular polarization (LHCP) whereas the right hand circular polarization (RHCP) indicates the clockwise rotation of the waves. Due to circular polarization (CP), the antenna always receives a component of the signal neglecting the orientation of 
the receiver. This is possible due to the resulting wave with an angular variation.

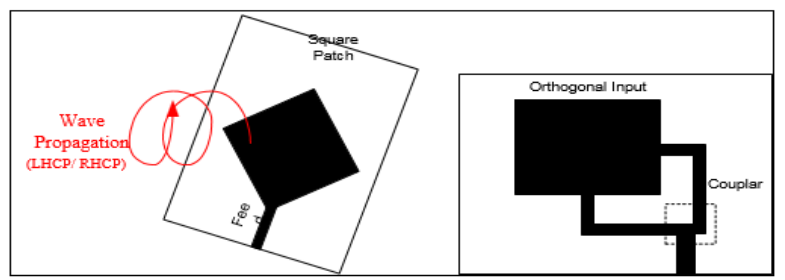

Fig 7.4 Circular polarization

\subsection{Bandwidth}

There are following definitions of bandwidth:

(a) The bandwidth is the frequency range over which the performance of the antenna is satisfactory.

(b) It is the frequency range over which it is matched with the feed line within required limits.

(c) The bandwidth of an antenna is the acceptable standing wave ratio (SWR) value over the concerned frequency range.

The channel have larger usable frequency range for more comfortable transmission.

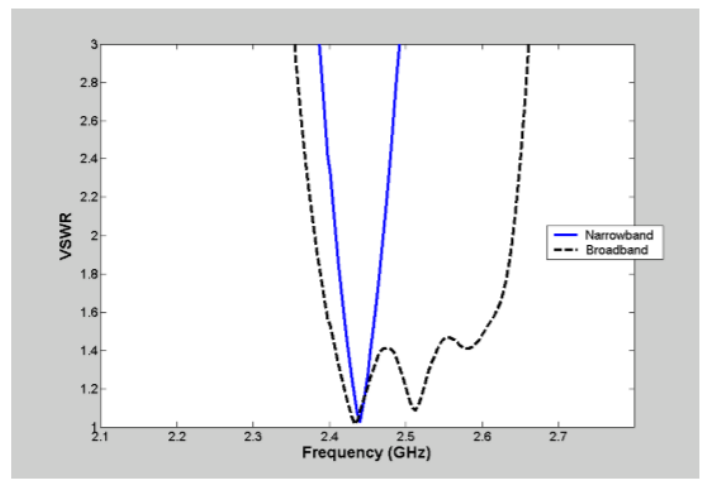

Fig 7.5 shows a typical broadband phenomenon in terms of frequency band usage

\section{FEEDING TECHNIQUES}

There are several feeding techniques of microstrip patch antennas. These methods are classified into following two categories:

1. Contacting method in which R.F. power is fed directly to the radiating patch using a connecting element like a microstrip line. Micro strip line and co-axial probe are main sub-categories of this method.

2. Non-contacting method:In this scheme, electromagnetic field coupling is applicable to transfer the power between the micro strip line and the radiating patch. The main subcategories of a non-contacting method are aperture coupling and proximity coupling.

\subsection{Microstrip Line Feed}

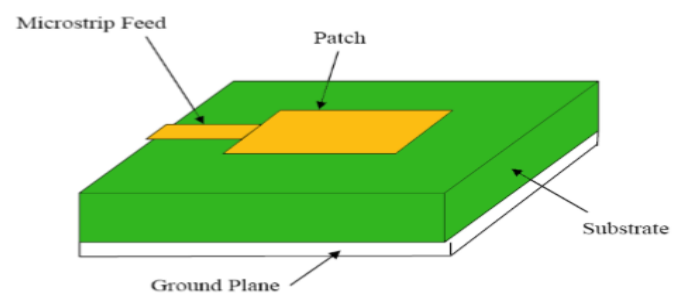

Fig 8.1 Microstrip line feed

In this technique, a conducting strip is directly connected to the edge of the microstrip patch. The conducting strip is smaller than the dimensions of the patch. The microstrip linefeed structures are suitable due to easy fabrication and lower cost. The feed may be etched on the same substrate to provide a planar structure.

The aim of this inset cut in the patch is to match the impedance of the feed line to the patch. In this case, there is no need of any additional matching element. This property is obtained by suitable inset position. If the thickness of dielectric substrate increases then the surface waves and spurious feed radiation increases. This hampers the banwidth of the antenna. The drawback of this feed structure is strong parasitic radiation

\subsection{Coaxial Feed}
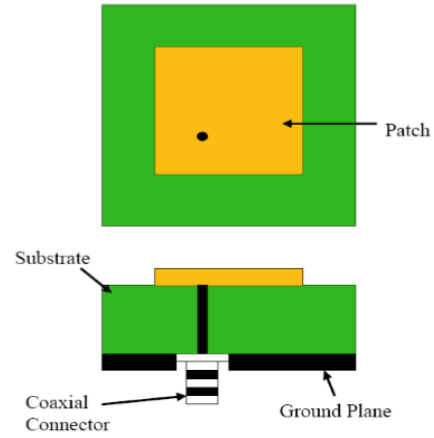

Fig 8.2 Coaxial feed rectangular microstrip patch antenna

The coaxial feed is one of common techniques for feeding of microstrip patch antennas. The inner conductor of the coaxial connector extends through the dielectric. In the case of connection of ground plane to the outer conductor, the inner conductor is soldered to the radiating patch. The functioning of coaxial feed is shown in figure (8.2)

There are following advantages of this feeding scheme:

1. The feeding may be placed at required location inside the patch to match its input impedance.

2. This method has low spurious radiation.

3. It is easy to fabricate.

The major disadvantages of coaxial feed are as following:

1. It provides narrow bandwidth

2. It is not easy to model because a hole is to be drilled in the substrate.

3. The connector protrudes outside the ground plane without making it completely planar to thick substrates $\left(\mathrm{h}>0.02 \lambda_{0}\right)$ 
It is remarkable to note that increased probe length for thicker substrates makes the input impedance more inductive which leads the matching problems. A thick dielectric substrate provides broad bandwidth. Several disadvantages of microstrip line feed and coaxial feed have been observed.

\subsection{Aperture Coupled Feed}

Some disadvantages of above mentioned feeding may be solved by this technique. In this feed technique, the ground plane separate the radiating patch and microstrip feed line.

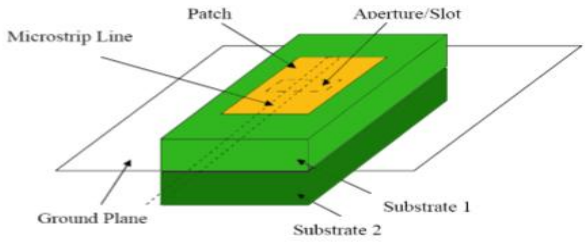

Fig 8.3 Aperture coupled feed

Coupling between the feed line and the patch is performed by a slot in the ground plane. Due to symmetry of the configuration, the coupling aperture is centered under the patch, above to lower cross polarization. The shape, size and location of the aperture are take into account for the amount of coupling from the feed line to the patch. Since the patch and the feed line are separated by the ground plane therefore the spurious radiation is minimum. A high dielectric material is useful for bottom substrate whereas a thick, low dielectric material is used for the top substrate to optimize radiation from the patch. The disadvantage of aperture coupled feed techniques are given below:

1. It is difficult to fabricate due to multiple layers.

2. It increases the thickness of the antenna.

3. This scheme provides narrow bandwidth.

\subsection{Proximity Coupled Feed}

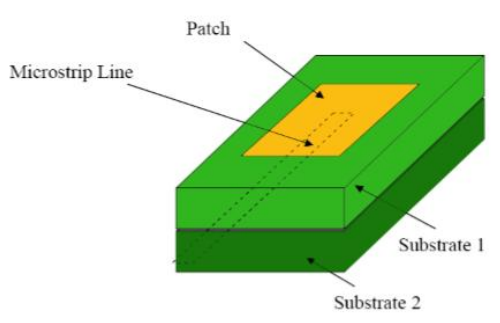

Fig 8.4 Proximity coupled feed

This technique belongs to electromagnetic coupling scheme. In this scheme, feed line is between two substrate. The radiating patch is situated on the top of the upper substrate. The main advantages of proximity coupled feed are as following:

1. It eliminates spurious feed radiation.

2. This technique provides very high bandwidth due to increase in the size of microstrip patch antenna.

3. There are choices between two different dielectric media where one for the feed line and other for patch to optimize the performance of the individual.

It is remarkable to note that matching is obtained by controlling the width-to-line ratio and the length of the feed line of the patch.

There are some disadvantages of this feeding scheme:
1. It is difficult to fabricate due to two dielectric layers which require suitable alignment.

2. There is an increase in the thickness of the antenna.

\section{SUBSTRATE PERMITTIVITY}

The dielectric constant is an important factor of the performance of the antenna. The width and the length are affected by it to alter resonant frequency and to reduce transmission efficiency. FR4 (G10 figerglass substrate) is used to determine permittivity $\left(€_{\mathrm{r}}\right)$. It alters from batch to batch. It also changes between different sheets of the substrate. The method of determining $\epsilon_{\mathrm{r}}$ are given below:

1. The Napoli-Hughes opened edged cavity method: A dielectric is used having copper on both sides of the substrate. The results show sharp spikes at resonant frequencies. The $€_{\text {eff }}$ is determined by this method. There is often no accuracy by this method.

2. The closed cavity method: The set up of this method is almost similar to Napoli-Hughes edged cavity method except the edges of the copper are short-circuited. In this process, the non-TEM waves propagate. This method has complication in its implementation.

3. The resonant line method: This method is easy in fabrication. It has ability to generate good approximations.

\section{DESIGN METHODOLOGY}

In order to find performance of the improvement of microstrip patch antenna, this paper has been split into subtask by which it can be completed. The flow chart makes easy to explain the process:

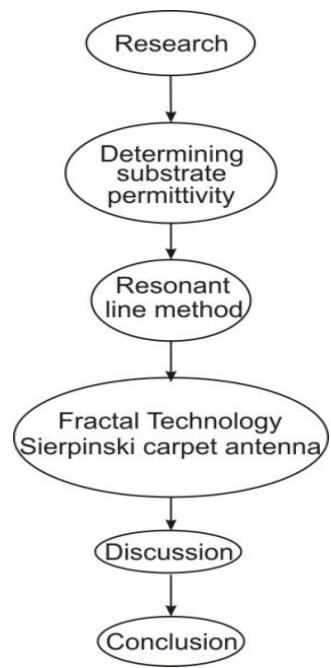

Fig 10.1 Design methodology

\section{DETERMINING SUBSTRATE PERMITTIVITY: RESONANT LINE METHOD}

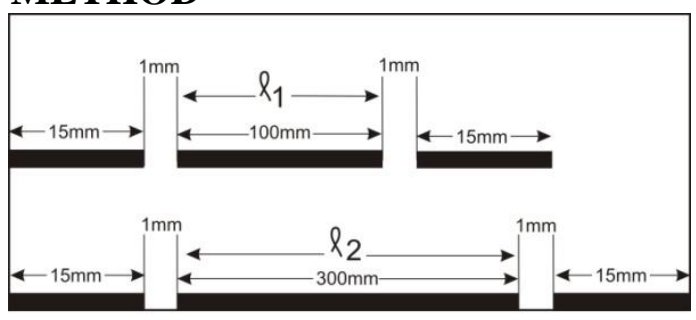

Fig. 11.1 
The value of dielectric constant $\varepsilon_{r}$ plays an important role in designing micro strip patch antenna. The Resonant line method is easy process. In this method two microstrip transmission line structures are used as shown in figure (11.1). It is important to note that when a signal is fed into the input port then the gaps work as open circuits and zero current is possible at these points. If the signal frequency has a wavelength of the form $1 \lambda / 2$, where 1 is the length of the centre section then the received signal may be maximum at the output port. The output is observed at peak when 1 is an integral multiple of $\lambda / 2$. The frequency of the signal is obtained using the network analyzer when the wavelength $\lambda$ is known. The length $l_{2}$ is thrice the size of $l_{1}$ to compensate to the effect of fringing at the ends. The formulae for dielectric constants $€_{\mathrm{r}}$ and $€_{\mathrm{eff}}$ are given below

$e_{r}=\left[\frac{c\left(n_{1} f_{2}-n_{2} f_{1}\right)}{2 f_{1} f_{2}\left(l_{2}-l_{1}\right)}\right]^{2}$

$e_{e f f}=\frac{\varepsilon_{r}+1}{2}+\left(\frac{\varepsilon_{r}-1}{2}\right)\left(1+12 \frac{h}{W}\right)^{\frac{-1}{2}}$

The width $\mathrm{W}$ of the microstrip patch antenna is calculated by

$$
W=\frac{c}{2 f_{r} \sqrt{\frac{\varepsilon_{r}+1}{2}}}
$$

\begin{tabular}{|c|c|c|c|}
\hline $\begin{array}{l}\text { Dielectric } \\
\text { constant } \\
€_{\mathrm{r}}\end{array}$ & Material & $\begin{array}{l}\text { Patch } \\
\text { length } \\
\mathrm{h}(\text { in } \\
\mathrm{mm})\end{array}$ & $\begin{array}{l}\text { Patch } \\
\text { width } \\
\text { W ( in } \\
\text { mm ) }\end{array}$ \\
\hline 2.1 & Teflon & 41.41 & 50.20 \\
\hline 3.2 & Taconic_TLC & 33.37 & 43.12 \\
\hline 4.4 & FR4 epoxy & 28.30 & 38.03 \\
\hline 5.7 & Mica & 24.72 & 34.14 \\
\hline 6.15 & Rogers R03006 & 23.76 & 33.05 \\
\hline 7 & Silicon_nitrate & 22.19 & 31.25 \\
\hline 8.3 & Marble & 20.28 & 28.98 \\
\hline 9.2 & Alumina 92pct & 19.20 & 27.67 \\
\hline 10 & Sapphire & 18.37 & 26.65 \\
\hline 11.9 & Silicon & 16.73 & 24.60 \\
\hline 12.9 & GallGallium_arseniium & 16.02 & 23.70 \\
\hline 16.9 & Diamond & 13.84 & 20.89 \\
\hline
\end{tabular}

This table shows that when dielectric constant is higher then dimensions of antenna are smaller

\section{HEIGHT OF DIELECTRIC SUBSTRATE(h)}

It is necessary that the microstrip patch antenna should not be bulky. Thus the height of the dielectric substrate should be small.

Here FR 4 epoxy substrate of standard height $1.59 \mathrm{~mm}$ is selected. Hence, the essential parameter for the design are:

- Frequency of operation $\mathrm{f}_{\mathrm{o}}=2.4 \mathrm{GHz}$

- Dielectric constant of substrate $€_{\mathrm{r}}=4.4$

- Height of dielectric substrate $\mathrm{h}=1.6 \mathrm{~mm}$

The transmission line model is used and $\mathrm{W}, \mathrm{e}_{\text {eff }}, \mathrm{L}_{\text {eff, }}, \Delta \mathrm{L}$ and $\mathrm{L}$ for required antenna are obtained by following formulae

\section{Width calculation $(\mathrm{W})$ :}

The width $\mathrm{W}$ of the Microstrip patch antenna is calculated as :

$$
W=\frac{c}{2 f_{r} \sqrt{\frac{\varepsilon_{r}+1}{2}}}
$$

Let $\mathrm{c}=$ free space velocity of light. Substit.uting $\mathrm{c}=3.0 \mathrm{e} 8 \mathrm{~m} / \mathrm{s}$, $\varepsilon_{r}=4.4$ and fo $=2.4 \mathrm{GHz}$, we get: $\mathrm{W}=0.03803 \mathrm{~m}=38.03$ $\mathrm{mm}$

2. Effective dielectric constant calculation $\left({ }^{e_{\text {reff }}}\right)$ The effective dielectric constant $e_{\text {reff }}$ is given by:

$e_{\text {reff }}=\frac{\varepsilon_{r}+1}{2}+\left(\frac{\varepsilon_{r}-1}{2}\right)\left(1+12 \frac{h}{W}\right)^{\frac{-1}{2}}$

Substituting : $\varepsilon_{r}=4.4, \mathrm{~W}=38.03 \mathrm{~mm}$ and $\mathrm{h}=1.6 \mathrm{~mm}, \mathrm{e}_{\text {reff }}$ $=4.3996$

\section{Effective length calculation ( Leff ):}

The effective length $L_{\text {eff }}$ is given by

$$
L_{e f f}=\frac{c}{2 f_{r} \sqrt{\varepsilon_{r_{e f f}}}}
$$

Substituting : $\varepsilon_{\text {reff }}=4.3996, \mathrm{c}=3.0 \mathrm{e} 8 \mathrm{~m} / \mathrm{s}$ and $\mathrm{f}_{\mathrm{r}}=2.4 \mathrm{GHz}$

$$
\mathrm{L}_{\text {eff }}=0.028569 \mathrm{~m}=28.569 \mathrm{~mm}
$$

4. Length extension calculation $(\Delta \mathrm{L})$ :

The length extension is $\Delta L$ given by,

$$
\Delta L=0.412 \frac{h\left(\varepsilon_{\text {eff }}+0.3\right)\left(\frac{W}{h}+0.264\right)}{\left(\varepsilon_{\text {reff }}-0.258\right)\left(\frac{W}{h}+0.8\right)}
$$

Substituting : $\varepsilon_{\text {reff }}=4.3996, \mathrm{~W}=38.03 \mathrm{~mm}$ and $\mathrm{h}=1.6 \mathrm{~mm}$, $\Delta \mathrm{L}=7.4800 \mathrm{e}-4=0.748 \mathrm{~mm}$

5. Actual length of patch calculation (L)

The actual length is obtained by: 
$\mathrm{L}=L_{\text {eff }}-2 \Delta \mathrm{L}$

Substituting $\mathrm{L}_{\mathrm{eff}}=4.3996 \mathrm{~mm}$ and $\Delta \mathrm{L}=0.7243 \mathrm{~mm}$,

$\mathrm{L}=28.30 \mathrm{~mm}$

\section{FRACTAL TECHNOLOGY}

Fractal technology is helpful in designing miniature antennas in which multiple band is integrated into a single device. It is notable that the objects having the character of self-similarity on all scales are fractals. Self-similarity and the fractal dimension are two important characters of a fractal. If the shapes of an object are similar on different scale then it is known as self-similar. There is a relation of the form

$L=N \times r$

Where $\mathrm{L}=$ estimated length of an object,

$\mathrm{N}=$ Number of rules needed to cover measured object,

$\mathrm{r}=$ length of the ruler,

If an object is reduced in Euclidian dimensions D and linear size $\frac{1}{r}$ in each spatial direction then increment in its measure is given by

$(\alpha N+\beta)=(\gamma r+\delta)^{D}, \alpha \geq 1, \gamma \geq 1, \beta \geq 0, \delta \geq 0$

Taking logarithmic of both sides of the above equation,

$\log (\alpha N+\beta)=D \log (\gamma r+\delta)$

Solving it,

$$
D=\frac{\log (\alpha N+\beta)}{\log (\gamma r+\delta)}
$$

This equation (13.2) is known as generalized Hausdorff dimension. If $\alpha=1, \gamma=1, \beta=0, \delta=0$ then the generalized Hausdorff dimension reduces to Hausdorff dimension as

$$
D=\frac{\log N}{\log r}
$$

The fractal antennas have the shape of fractal structures. In fractal antennas, the shapes are repeated and each of shapes has unique attributes. Sierpinski gasket, Kochi island, Sierpinkski carpet, Minkowski and Hilbert curve are different shapes of fractal antennas. In this section, microstrip Sierpinski carpet antenna has been studied.

In initial design, the square microstrip patch antenna is considered. The microstrip patch antenna design equation is helpful in determining its dimensions. In case of Sierpinski carpet antenna, Transmission line feeding technique is chosen. The figure of next page shows the iteration of the microstrip Sierpinski carpet fractal antenna upto $3^{\text {rd }}$ iteration.
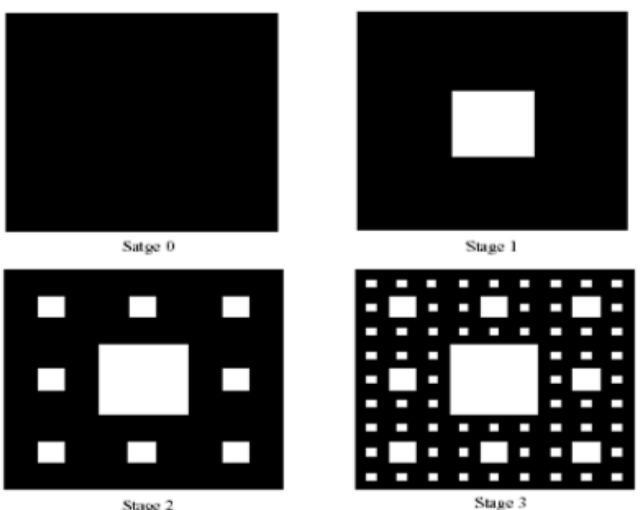

Fig 13.1 Sierpinski carpet square antenna with third iteration

Suppose

$\mathrm{N}_{\mathrm{n}}=$ The number of black boxes,

$\mathrm{L}_{\mathrm{n}}=$ The ratio for the length, iteration,

$A_{n}=$ The ratio for the fractional area after the $n^{\text {th }}$

$\mathrm{d}_{\mathrm{n}}=$ The capacity dimension,

Let $\quad \mathrm{N}=16^{\mathrm{n}}$

$$
\begin{gathered}
L_{n}=\left(\frac{1}{6}\right)^{n}, A_{n}=\left(\frac{16}{17}\right)^{n} \\
d_{n}=-\lim _{n \rightarrow \infty} \frac{\ln N_{n}}{\ln L_{n}}=-\lim _{n \rightarrow \infty} \frac{n \ln 16}{n \ln \left(\frac{1}{6}\right)} \\
=\frac{\ln 16}{\ln 6}=\frac{2.77259}{1.79176}=1.54741148
\end{gathered}
$$

Table: Fractal antenna properties

\begin{tabular}{|l|c|c|c|c|}
\hline & $\begin{array}{l}\text { Zero } \\
\text { iteration }\end{array}$ & \multicolumn{1}{c|}{$\begin{array}{l}1^{\text {st }} \\
\text { iteration }\end{array}$} & $\begin{array}{l}2^{\text {nd }} \\
\text { iteration }\end{array}$ & $\begin{array}{l}3^{\text {rd }} \\
\text { iteration }\end{array}$ \\
\hline $\mathrm{N}_{\mathrm{n}}$ & 1 & 16 & 256 & 4096 \\
\hline $\mathrm{L}_{\mathrm{n}}$ & 1 & 0.1667 & 0.0278 & 0.0046 \\
\hline $\mathrm{A}_{\mathrm{n}}$ & 1 & 0.9412 & 0.8858 & 0.83337 \\
\hline
\end{tabular}




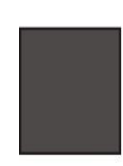

(A)

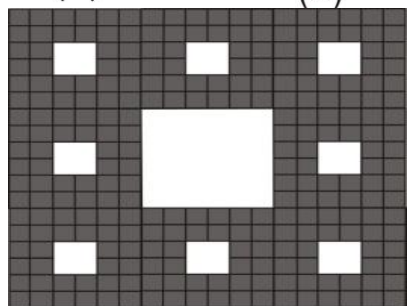

(C)

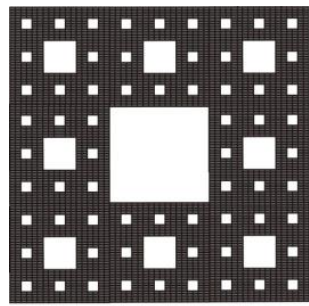

(D)

Figure (13.2) Microstrip Sierpinski Carpet Antenna width transmission line feeding of (a) zero iteration (b) First iteration (c) Second iteration (d) Third iteration

\subsection{Return Loss Measurement}



Fig 13.1.(1) Measurement and simulation of return loss for fractal antenna

Fig 13.1.1 shows the measurement and simulation result of input return loss which is concerned with Sierpinski carpet with third iteration. For the $1^{\text {st }}$ band upto the $3^{\text {rd }}$ band, the bandwidth is between 2.5 to $5.5 \%$. At the frequency $5.3 \mathrm{GHz}$ the $4^{\text {th }}$ band the bandwidth is $13.6 \%$ and at band five for frequency $7.96 \mathrm{GHz}$, the bandwidth is increased to $4.8 \%$.

\subsection{Radiation Pattern}

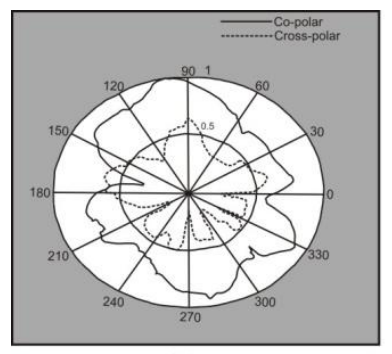

(a)

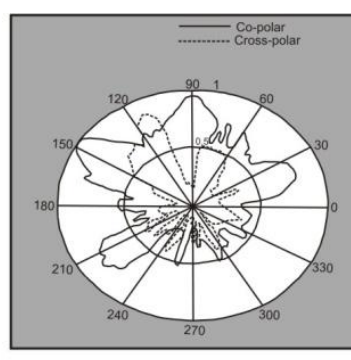

(b)
Fig 13.2 (1): Co and cross polar radiation pattern for microstrip sierpinski carpet antenna for (i) $2.78 \mathrm{GHz}$ and (ii) $5.3 \mathrm{GHz}$

An anechoic chamber is suitable for the measurement of the far-field radiation pattern. The figure 13.2(1) shows co-polar and cross-polar radiation pattern at the first and four band. Due to geometrical asymmetry of the fractal structure in E plane with respect to the feed point, there is multiple lobes of the radiation pattern at a higher band.

\subsection{Discussions}

In the study of Sierpinski carpet antenna, following points have been considered for further investigation.

1. The measurement of the return loss depends on the trend of the simulation process.

2. Considering the momentum simulation from the microwave office software, in this antenna the simulation is carried out.

3. This antenna gives a multiple frequency for three iterations.

4. In general, the resonance frequencies initiate from 2.78 $\mathrm{GHz}$ having a return loss of $-16.55 \mathrm{~dB}$.

5. If the higher frequency is at $3.56 \mathrm{GHz}$ then there is a return loss of $-11.9 \mathrm{~dB}$.

6. At the higher frequency, the bandwidth is increased up to $48 \%$.

7. The radiation pattern is remarkable for E-plane for two different frequencies.

8. The cross polar isolations are $15.5 \mathrm{~dB}$ and $10.5 \mathrm{~dB}$ at 2.78 $\mathrm{GHz}$ and $5.3 \mathrm{GHz}$ frequency respectively.

\section{CONCLUSIONS}

By this study, following conclusions are derived:

1. By introducing defected shape in ground plane, the consumers suffer by the drawback of rectangular microstrip patch antenna (RMPA).

2. Defected ground plane is used for improving the bandwidth. This is the main drawback of the patch antenna.

3. By achieving the improvement of bandwidth up to $34.1846 \mathrm{MHz}$, the antenna may be used for wide band applications.

4. Reduced reflection coefficient shows that the maximum power is radiated from the patch and a small amount of reflection waves is returned back to the source.

5. By the help of different shapes, dimensions and locations of defected ground structure (DGS), the improvements in parameters may be achieved as per need of the society in wireless communication.

6. In case of square microstrip Sierpinski carpet antenna, the antenna is constructed by fractal geometry for multiband operation. This antenna indicates a good radiation and input return loss.

7. Sierpinski carpet antenna is best possible antenna for wireless communications for multiband operation.

\section{AKNOWLEDGEMENT}

Authors are grateful to referee for his valuable suggestions and comments which improve the presentation of this paper.

\section{REFERENCES}

[1] Sakshi Kapoor, Davinder Parkash, Efficient Microstrip Fed Rectangular Patch Antenna with DGS for WLAN \& 
WiMAX Applications, IJERA Vol. 2, Issue 6, November - December 2012, pp.044-047.

[2] Bharti Gupta, Sangeeta Nakhate, Madhu Shandilya, Compact Ultera Wideband Microstrip Antenna with Modified Ground Plane for Bandwidth Enhancement, International Journal of Computer Applications, Volume 49-No.19, July 2012, (0975 - 8887).

[3] Ashwini K.Arya, M. V. Kartikeyan, A Patnaik, Defected Ground Structurein the perspective of Microstrip antenna, requenz, Vol. 64, Issue 5-6, pp.79-84, Oct- 2010

[4] Neha Ahuja, Rajesh Khanna, Jaswinder Kaur, Dual Band Deffected Ground Microstrip Patch Antenna for WLAN /
WiMax and Satellite Application, International Journal of Computer Applications, Volume 48 - No.22, June 2012.

[5] K. Dinakaran , M. Vajikabanu , M. Piriyadharsini , D. Rajeshwari,, Design of Microstrip Patch Antenna For Wi$\mathrm{Fi}$ Applications International Journal of Advanced Research in Electronics and Communicatin Engineering(IJARECE), Volume 5, Issue 1, January 2016.

[6] Avni Kapadia, Kanu Patel, Design and Analysis of Various Parameter of Patch Antenna Using Fractal Geometries, Volume 5, Issue 3, March 2016. 